\title{
Fuzzy Logic Approach for Person Authentication Based on Palm-print
}

\author{
Rajkumar Mehar ${ }^{1}$, Kapil Kumar Nagwanshi \\ ${ }^{1}$ Computer Science \& Engineering Department, Rungta College of Engineering \& Technology, \\ Kohka Kurud Road, Bhilai \\ ${ }^{1}$ rajmeharmehar24@gmail.com, ${ }^{2}$ kapilkn@ieee.org
}

\begin{abstract}
The aim of present research is to classify the palmprint images using fuzzy logic for different security aspects. Fuzzy logic is relatively efficient and advanced theory, at present wide use of fuzzy logic is in the classification of remotely sensed images. A branch of biometric, palmprint authentication have increasing attention because palmprint is unique, permanent, measurable characteristics having voluminous of the line features. In this paper we discuss a method for feature extraction, identification (recognition) techniques of palmprints based on fuzzy logic technique and some publically available databases. This method use sub image based principle line feature extraction technique in low resolution palmprint images. Image is divided into sub images and feature obtained from these subimages are combined to generate a single feature vector for the palmprint image. This vector is provides to fuzzy inference system as input. The testing for system has been performed on IITD, and PolyU databases. Experiments were carried to show the effectualness of our proposed approach have an accuracy of $89.46 \%$.
\end{abstract}

Keywords: Biometric, ROI, Fuzzy Inference System, Membership functions, if-then rules.

\section{INTRODUCTION}

For security system, the authentication and identification of a person is carry out with signature, user id, password and cards. These techniques are not enough at present days because signature can be replicated, passwords can be guessed and cards can be misplaced or stolen So, biometric features are used to recognize an individual. The recognition of an individual is performed via two steps identification (validation) and authentication (verification) process. The biometric features are consists of behavioural characteristics (heart-beat, voice) and anatomical (fingerprints, face, palm, iris) [1]. The palmprint is best for the recognition of an individual. Palmprint is a pattern of principle lines ridges wrinkles and minutie points. Each and every individual has its unique and different palmprint. In this paper we discuss about image segmentation, feature extraction, identification and verification (recognition) techniques and some publically available databases. 


\section{IMAGE PREPROCESSING AND SEGMENTATION}

Preprocessing is the procedure to align palmprint position and segment the central area for feature generation. Palmprint segmentation is an important pre-processing step in automatic biometric authentication system. Researchers use four different types of sensors: digital scanners, digital cameras, video cameras and CCD based scanners to gather palmprint images. CCD based palmprint scanner aligns palm accurately and scan biometric traits to collect high quality images. Scanners based on CCD employs pegs to restrict palm orientation for guiding the position of hands [2]. The problem arises in contactless scanning is image quality as image quality is low, cause recognition problem. To establish coordinate system major techniques utilize the key points in between fingers. Preprocessing involve five common processes: (1) palm print images binarization, (2) extracting the contour of palmprint, (3) key point detection, (4) coordination system establishment and (5) ROI extraction, the central part of image [3]. Fig 1 (h) shows a preprocessed image. Majority of the preprocessing algorithms select ROI of square area but few of them select ROI of half elliptical and circular area for feature extraction. The square shaped region of extracted ROI is easy to handle translation operation. Half elliptical and circular shaped ROI are easy to handle rotation to the palmprint image.

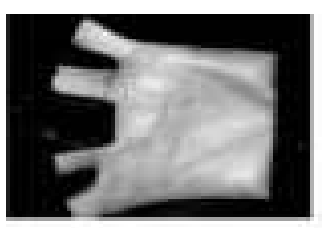

a

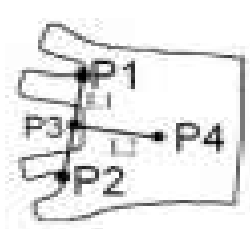

e

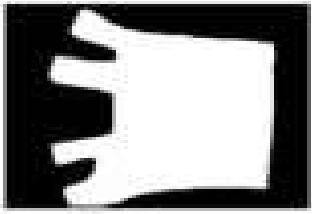

b

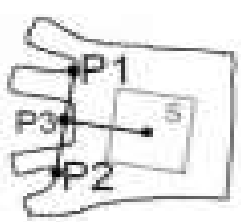

$f$

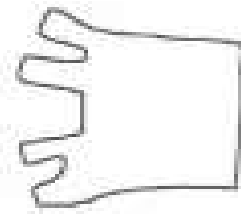

C

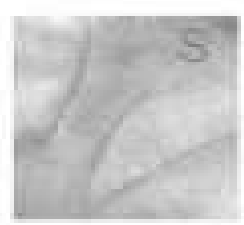

g

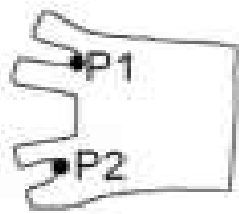

d

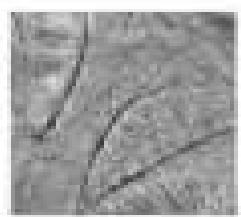

h

Figure 1: Palmprint Image Peprocessing. (a) Original Image, (b) Image Binarization, (c) Boundry Tracking, (d) Key Point Detection, (e) Establish Coordinate System, (f) Select Central Area, (g)segmented ROI (h) ROI Extracted Image.

\section{FUZZY INTERFERENCE SYSTEM}

Fuzzy inference is the process, using fuzzy logic to formulate the mapping from a given input to an output [1]. The fuzzy inference process involves three main factors membership functions, if-then rules and fuzzy logic operators. Image processing in fuzzy inference system (FIS) is consecutive task of Image fuzzification, membership functions \& rules creation and image defuzzification. There are two types of fuzzy inference systems that can be implemented in the Fuzzy Logic Toolbox:

- Sugeno-type. 
- Mamdani-type.

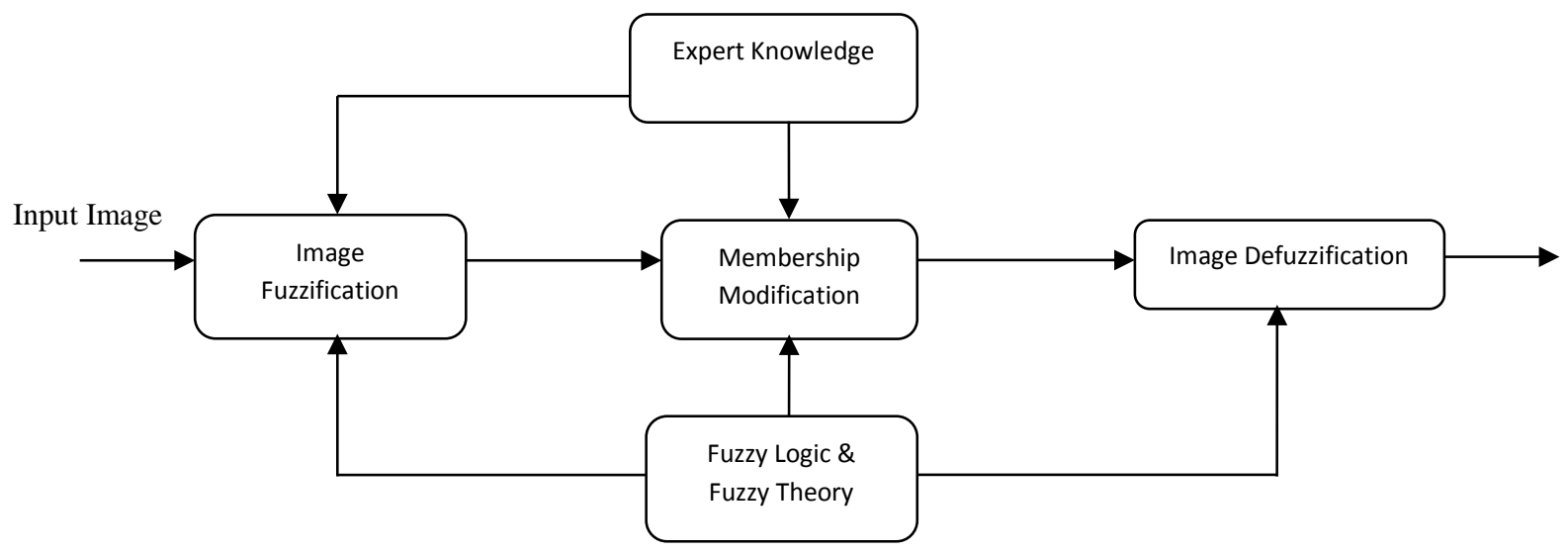

Figure 2: Fuzzy Inference System

Mamdani type fuzzy inference method expects the output membership functions are to be a fuzzy set. It is the most commonly used fuzzy methodology. For each output variable there is a fuzzy set, necessary to aggregate and defuzzified as shown in above figure 2 [4]. A Sugenotype system is useful to model an inference system having the output membership functions are either linear or constant. Fuzzy inference system of sugeno type is used in recognition in this paper. There are 100 inputs to the fuzzy inference system having range from 0 to 255 . One output variable is designed with range from 0 to 500. Image is segmented into 100 blocks, generates one feature value. These feature value of all blocks is considered as input to each input variable. Input variable consist a number of membership function of triangular function (trimf), all membership function is same type. Output variable also have membership function of constant type one for each class. FIS consist 100 rules for recognition purpose by setting up rule weight to 1 and using AND fuzzy operator to connect antecedent of the rule. Figure 3 shows the rule editor of FIS system.

Necessity of FIP: there are many reasons for use of fuzzy techniques in image processing. The most important is in many image-processing applications, it is necessary to use expert knowledge to avoid the difficulties (e.g. scene analysis, object recognition). Fuzzy logic and Fuzzy set theory provides us a powerful tool to represent the human knowledge in the form of fuzzy if-then rules. On the other side due to uncertain data, many difficulties in image processing arise. However, this uncertainty is not always due to randomness of data but to the ambiguity and vagueness of data. 


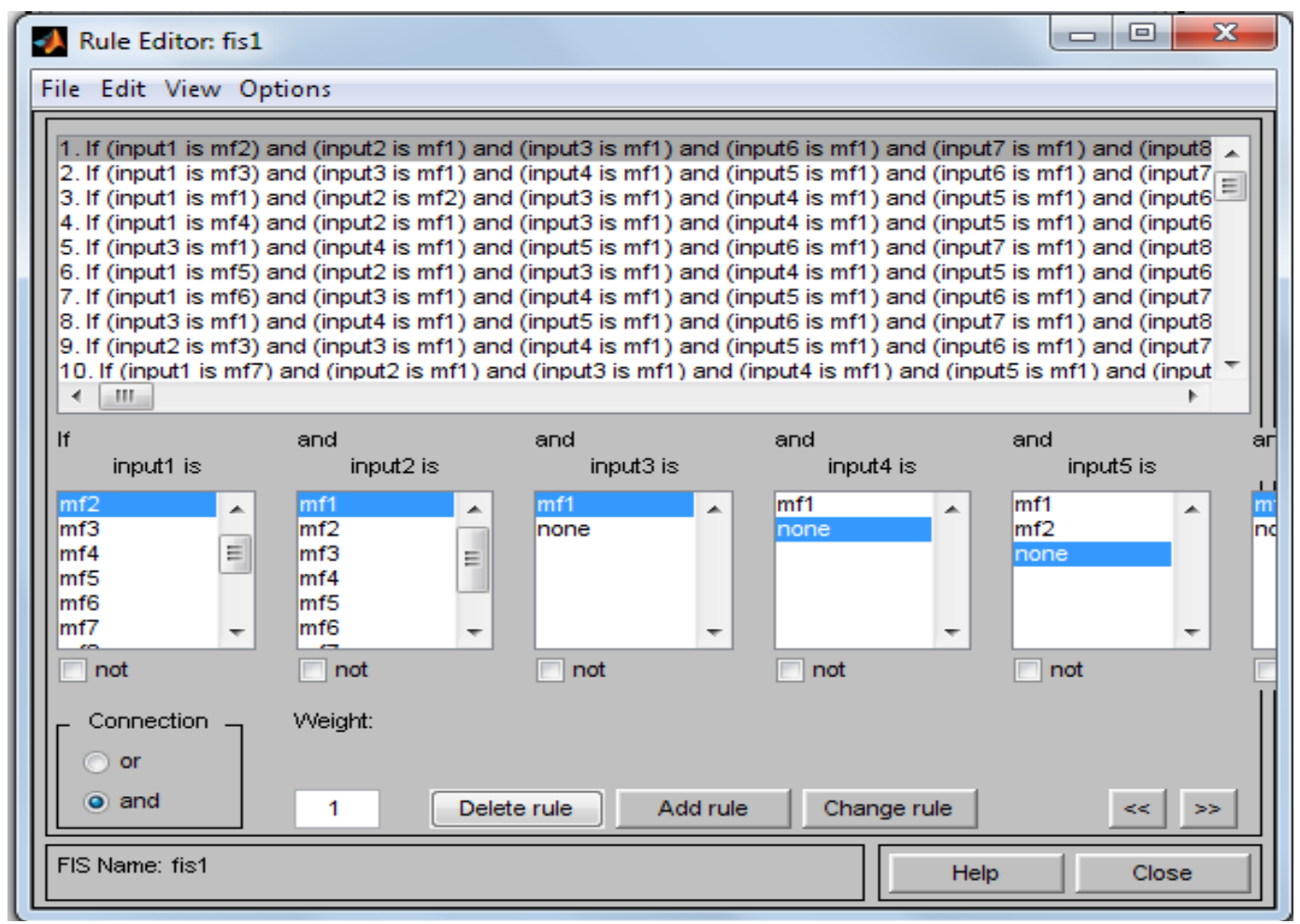

Figure 3: Sample of the Fuzzy Logic Rules Editor

\section{FEATURE EXTRACTION}

Once the central part is segmented in preprocessing, next step is to extract features for matching operation. Two different types of algorithms are used for recognition, verification and identification. Typical algorithms for Verification are line based, subspace based and statistic based. Line-based approach either uses an existing edge detection method or develops an edge detection method to extract palm line $[9,10]$. These line are matched either directly or represented in other formats for matching.

Canny edge detector [5] used to detect palm line as shown in figure 4 (b) below. The orientation of the edge point is passed into four membership function representing four directions and finally Euclidian distance is used for pattern matching.

In Subspace-based methods also called appearance based methods in the literature of the recognition. They use principal component analysis (PCA), linear discriminant analysis (LDA), and independent component analysis (ICA) [11]. The subspace coefficient is regarded as features. In subspace method various distance measure and classifier are used to compare the features. In addition to apply PCA, LDA, and ICA directly to palmprint images, researchers also use wavelets [12], Gabor, discrete cosine transform (DCT) and kernel in their method.

Statistical based methods are either local or global statistical approaches. Local statistical approaches transform images into another domain and then divide the transformed images are divided into several small regions. Local statistics for example means and variances of each small region are calculated and regarded as features. To our survey knowledge, no one has yet 
investigated high order statistics for these approaches. The small regions are commonly square but some are elliptical and circular.

In this paper image is filtered for edge detection and then segmented into small regions known as blocks, fig 4 (c) demonstrates the segmentation of palmprint image. After image filtering each point has an intensity value greater than 0 if it is a line element otherwise intensity value is zero.

Feature extraction algorithm is as described below:

- Filter the image with Canny Edge Detection Filter to detect the principle lines with a threshold value.

- Segment the filtered image into sub images (blocks) and Find the no of line element in each block.

- Extract one feature from each block by finding the average intensity value of pixels which is a line element.

- Then features from all sub images are aggregated into a single string to generate feature vector of the image.

Image have different resolution in different dataset so, at first images are resized to $150 * 150$ resolution then segmented to 100 blocks of $15 * 15$ resolution.

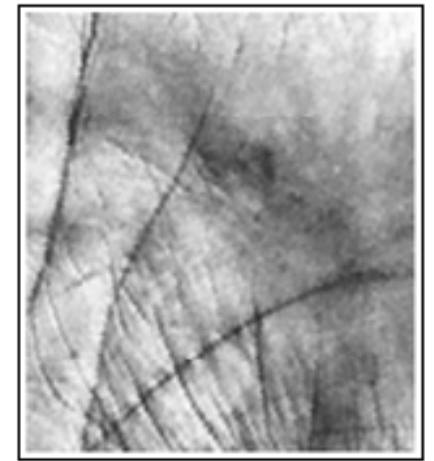

(a)

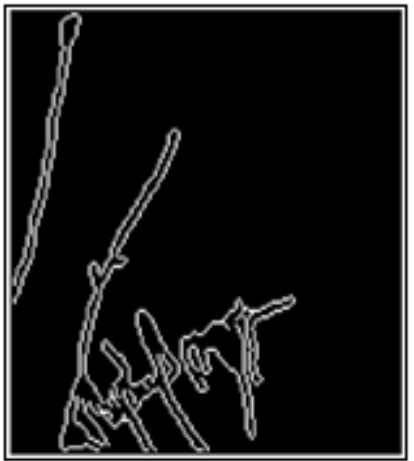

(b)

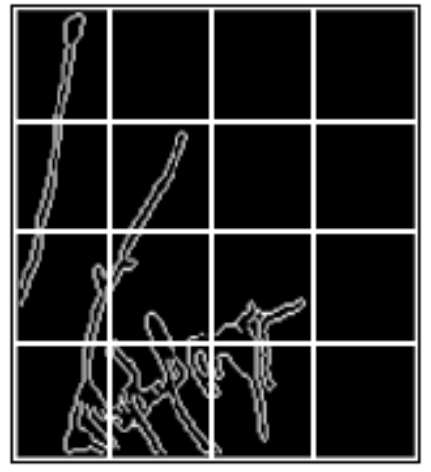

(c)

\begin{tabular}{|c|c|c|c|}
\hline V1 & V2 & $\cdots$ & V10 \\
\hline V11 & V12 & $\cdots$ & V20 \\
\hline$\cdots$ & $\cdots$ & $\cdots$ & $\cdots$ \\
\hline V91 & V92 & $\cdots$ & V100 \\
\hline
\end{tabular}

(d)
$[\mathrm{V} 1, \mathrm{~V} 2, \mathrm{~V} 3, \mathrm{~V} 4, \mathrm{~V} 5, \ldots \ldots . . \mathrm{V} 100]$

(e)

Figure 4: Feature Extraction. (a) Palmprint Image, (b) Palmline Detection using Canny Filter, (c) Segmentation of Image, (d) Average Intensity Value of Each Blocks, (e) Feature Vectore of image. 


\section{$5 \quad$ RECOGNITION}

The process of image matching or classification is to compute the degree of similarity between the input test image and a training image from database. In this project the main purpose of fuzzy logic is to perform classification task. For recognition purpose lots of different classifiers have been designed alike SVM, Neural Network (NN), KNN, GMM, LDA, HMM. Fuzzy classification is performed effectively by using and modifying two component, Membership function and Fuzzy logic rules. Fuzzy rules are in the form of "if then else".

If $\mathrm{X} 1$ is $\mathrm{A} 1$ and $\mathrm{X} 2$ is $A 2$ Then $\mathrm{Y}$ is $\mathrm{B}$.

Where $\mathrm{X} 1$ and $\mathrm{X} 2$ are fuzzy variables and $\mathrm{A} 1$ and $\mathrm{A} 2$ are fuzzy values. The if part of the rule "X1 is $A 1$ " is known as premise or antecedent, and then part of the rule "Y is $B$ " is known as conclusion or consequent. Statements in the antecedent (or consequent) section of the rules can employ fuzzy logical connective such as "OR" and "AND". In the if-then rules, the word "is" have used in entirely two dissimilar ways depends on whether it comes alongs in the antecedent part or in the consequent part.

The image used for testing is either enrolled or not. If image is enrolled then output is a value from 1 to 100 because 100 rules are created. Otherwise image does not belong to any rule and output value is mid range of output variable. For example if image is of second class then rule 2 is executed and output value is 2 . If image is not a genuine image then output value is 250 as range of output variable is 0 to 500. Fuzzy rule consists 103 columns 100 for input variables to take input from each block, 1 for output variable to generate the output value, 1 for fuzzy operator which is set to 1 for AND fuzzy operator and last column for fuzzy rule weight range from 0 to 1 , here rule weight is set to 1 .

\section{AVAILABLE DATABASE}

Three palmprint image databases are publicly available for the research purpose; these databases are namely CASIA palmprint database, PolyU palmprint database and IIT Delhi palmprint database. The CASIA palmprint image database consist total 5502 palmprint images of 312 subjects [6]. For each person, the left and right palm images have been captured. All the palmprint images have been captured by their self developed "palmprint recognition device". These palm images are in JPEG format of 8 bit gray level files. This self developed device does not have any pegs to restrain the postures and positions of the palmprints.

PolyU palmprint database consists 500 different palms with 12 samples for one illumination and thus having total 6000 images [7]. The images were accumulated from 55 females and 195 males in two different academic sessions. The images are collected from the person having age between 20 to 60 years. In both sessions, 6 palmprint samples are taken for every palm. PolyU database utilize pegs to avoid the hand orientation. Therefore, this database is used widely as utilization of pegs helps to acquire significantly higher performance. 
IIT Delhi palmprint dataset is developed from the image of staff member and students of IIT Delhi during Jul 2006 to Jun 2007 [8]. IITD dataset uses a touch less image acquisition setup. Database consists 235 users palm image, is stored in bitmap ( ${ }^{*}$. bmp) format. For each person 7 images are captured of both left and right hand palm in different hand variation. The touch less imaging results in higher image scale variations. The captured images have $800 \times 600$ pixels resolution, segmented or cropped image in normalised form of $150 \times 150$ resolutions are also available. In this paper we use four dataset, IIT DELHI Palmprint database of Left Hand is dataset1, IIT DELHI database of Right Hand is dataset2, PolyU 2D+3D Palmprint Database is dataset3, PolyU Multispectral Palmprint Database of Blue Illusion is dataset4. For testing process 225 images are taken from dataset1 470 images from dataset2, 570 images from dataset 3 and from dataset4 of blue illumination 792 images are used.

\section{$7 \quad$ RESULT \& DISCUSSION}

User interface of proposed system is shown in figure 5. At first palm print recognition system is trained by selecting the training data from any dataset as in figure 6 (a). Thereafter fuzzy inference system is designed on the basis of features extracted from the training dataset. Thereafter testing data is selected to test the system accuracy from corresponding dataset. Figure 6 (b) shows the testing of data to the system. Based on the methodology four dataset are tested in different threshold value and results are obtained as shown in table1. It is cleared from table1 that dataset 2 have the highest accuracy 89.46 and also in different threshold value dataset 2 have best result. Figure7 show the line graph of accuracy to all four dataset at different threshold value. Table 2 shows the comparison of template size in byte of different methods. System speed of various methods in dataset1 is shown in table 3(a) and system speed of various methods in PolyU palmprint dataset is shown in table 3 (b).

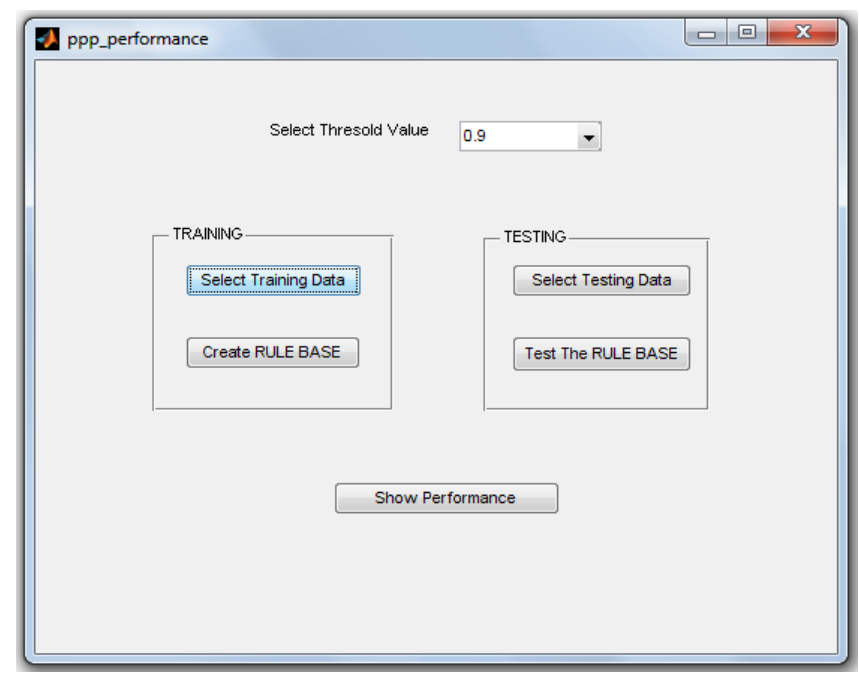

Figure 5: GUI of Palmprint Recognition System 


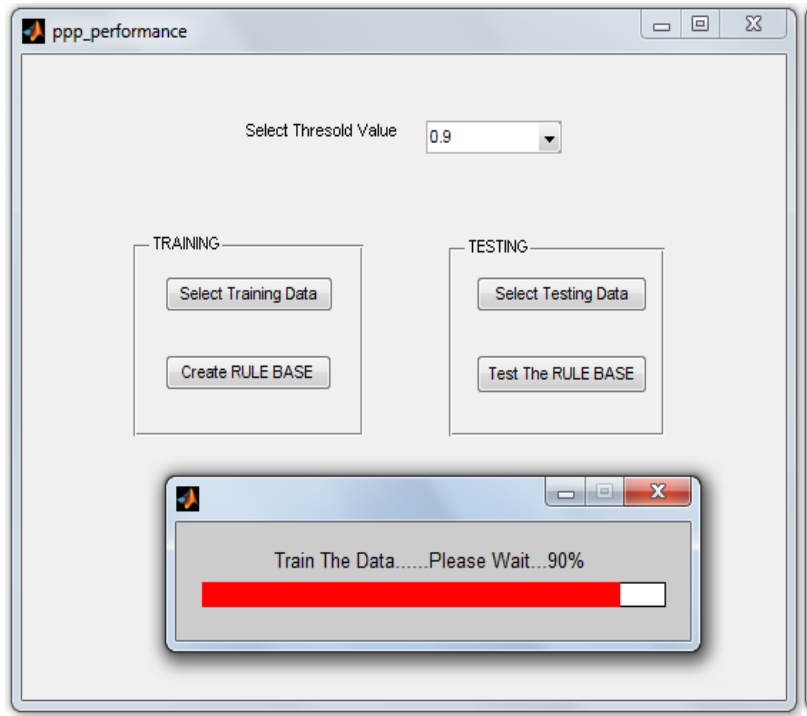

(a)

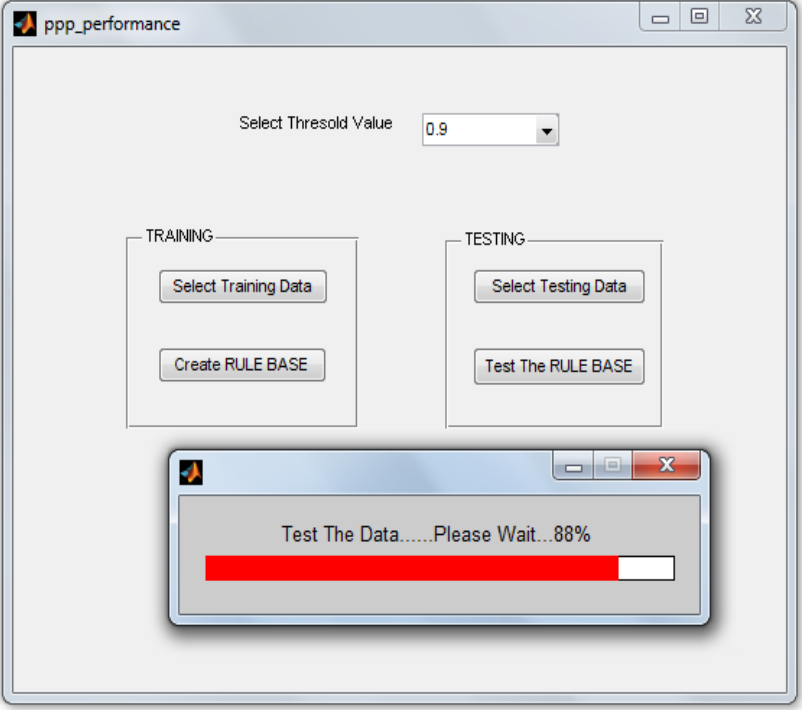

(b)

Figure 6: Identification and Verification process (a) Training to the system, (b) Testing to the system.

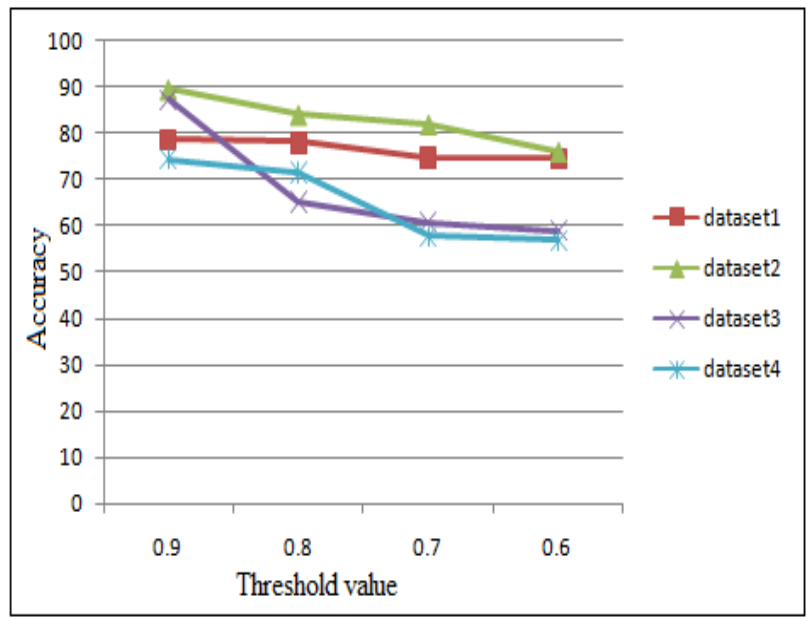

Figure 7: Accuracy of the different datasets

Table 1: Verification accuracies of different databases

\begin{tabular}{|c|c|c|c|c|}
\hline Threshold value & Dataset1 & Dataset2 & Dataset3 & Dataset4 \\
\hline 0.9 & 78.6 & 89.46 & 87.19 & 74.30 \\
\hline 0.8 & 78.0 & 83.93 & 65.08 & 61.48 \\
\hline 0.7 & 74.88 & 81.80 & 60.78 & 57.57 \\
\hline 0.6 & 74.67 & 75.85 & 58.94 & 56.88 \\
\hline
\end{tabular}

Table 2: comparison of template size

\begin{tabular}{|l|c|}
\hline \multicolumn{1}{|c|}{ Method } & Template size (byte) \\
\hline Competitive code & 384 \\
\hline Palm code [13] & 256 \\
\hline 2D SAX [17] & 256 \\
\hline This paper & 100 \\
\hline
\end{tabular}




\section{Table 3: comparison of speed}

(a) System speed for dataset 1

\begin{tabular}{|l|l|l|l|}
\hline \multicolumn{1}{|c|}{ Method } & \multicolumn{1}{c|}{ Extraction } & \multicolumn{1}{c|}{ Matching } & \multicolumn{1}{c|}{ Total } \\
\hline Palmcode [13] & 336.1316 & 2.7186 & 338.8502 \\
\hline Ordinal code [14] & 672.9061 & 7.9563 & 680.8624 \\
\hline Kipsang [15] & 15.813 & 1.4971 & 17.3101 \\
\hline Dale et al [16] & 12.343 & 0.0100 & 15.3530 \\
\hline This paper & 69.9391 & 67.6674 & 137.6089 \\
\hline
\end{tabular}

(b) System speed for PolyU palmprint dataset

\begin{tabular}{|l|l|l|l|}
\hline \multicolumn{1}{|c|}{ Method } & \multicolumn{1}{c|}{ Extraction } & \multicolumn{1}{c|}{ Matching } & \multicolumn{1}{c|}{ Total } \\
\hline Palmcode [13] & 54.4166 & 0.73156 & 55.1482 \\
\hline Ordinal code [14] & 155.1370 & 2.3652 & 157.5022 \\
\hline Kipsang [15] & 14.4190 & 0.4813 & 14.9003 \\
\hline Dale et al [16] & 5.3010 & 0.0100 & 5.3110 \\
\hline This paper & 288.9478 & 201.1187 & 490.0753 \\
\hline
\end{tabular}

\section{CONCLUSION}

Salient features of the proposed technique include a low resolution image for feature representation of the palmprint texture, low computational overheads having recognition accuracies over $89 \%$. Accuracies held over 100 classes having 5 samples per class have shown it to be an acceptable and optimized method providing comparable recognition efficiency. Future work would involve testing on more number of classes and fusion of other biometric features like colour of palm, face, or other biometric trait to improve recognition accuracies. This paper also does not include any preprocessing steps i.e. the images are taken from the publicly available dataset as it is.

\section{REFERENCES}

[1]. Davide Maltoni, Dario Maio, Anil K. Jain, Salil Prabhakar, Handbook of Fingerprint Recognition, Springer Press 2nd Edition 2009.

[2]. C. C. Han, $A$ hand-based personal authentication using a coarse-to-fine strategy, Image and Vision Computing, 2004. 22 (11): p. 909-918.

[3]. S. S. Hatkar, Sneha M. Ramteke, Segmentation of Palmprint into Region of Interest, IJCT ISSN 2277-3061, March-April 2013. 4(2).

[4]. G. Prasanna Lakshmi, J. A. Chandulal, Y. Gopala Krishna, Finger print Analysis and Matching using fuzzy logic design, IOSR Journal of Computer Engineering (IOSRJCE) ISSN: 2278-0661, July-Aug 2012. 1(6): p. 0408. 
[5]. J. Canny, "A computational approach to edge detection, IEEE Transactions on Pattern Analysis and Machine Intelligence, 1986. 8(6): p. 450-463.

[6]. CASIA Palmprint Database, http://biometrics.idealtest.org/dbDetailForUser.do?id=5.

[7]. PolyU Palmprint Database, "http://www.comp.polyu.edu.hk/biometrics".

[8]. IITD Touchless Palmprint Database. Version 1.0 (available online): http://web.iitd.ac.in/ ajaykr/Database Palm".htm. http://www4.comp.polyu.edu.hk/ csajaykr/IITD/Database Palm.htm.

[9]. D.S. Huang, W. Jia, D. Zhang, Palmprint verification based on principal lines, Pattern Recognition, 2008. 41 (4): p. 1316-1328.

[10]. M.K.H. Leung, A.C.M. Fong, H.S. Cheung, Palmprint verification for controlling access to shared computing resources, IEEE Pervasive Computing, 2007. 6 (4): p. 40-47.

[11]. M.K.H. Leung, A.C.M. Fong, H.S. Cheung, Palmprint verification for controlling access to shared computing resources, IEEE Pervasive Computing, 2007. 6 (4): p. 40-47.

[12]. M. Ekinci, M. Aykut, Palmprint recognition by applying wavelet subband representation and kernel PCA, Lecture Notes in Artificial Intelligence, 2007. p. 628-642.

[13]. D. Zhang, A. W. Kong, J. You, M. Wong, Online palmprint identification, IEEE Transactions on Pattern Analysis and Machine Intelligence, 2003. 25(9): p.1041-1050.

[14]. Z. Sun, T. Tan, Y. Wang, Ordinal palmprint representation for personal identification, in: Computer Vision and Pattern Recognition, 2005. p. 279-284.

[15]. H. K. Choge, T. Oyama, S. Karungaru, S. Tsuge, M. Fukumi, Palm- print recognition based on local dct feature extraction, in: International Conference on Neural Information Processing, 2009. p. 639-648.

[16]. M. P. Dale, M. A. Joshi, N. Gilda, Texture based palmprint identification using dct features, International Conference on Advances in Pattern Recognition, 2009. pp. 221-224.

[17]. Jiansheng Chen, Yiu-Sang Moon, Ming-Fai Wong, Guangda Su, Palmprint authentication using a symbolic representation of images, Image and Vision Computing, 2010. 28: p.343-351. 